Editorial

\title{
The long quest for cancer cures
}

Volume 6 Issue 2 - 2016

\section{Editorial}

The quest to battle cancer began some 4,000years ago. Indeed, cancer is one of the oldest diseases seen in human specimens-quite possibly the oldest. It is also an age-related disease. However, it was fleetingly rare because hidden by other illnesses: cholera, dropsy, leprosy, plague, pneumonia, smallpox and tuberculosis. As these diseases were vanquished, and as the human lifespan lengthens, cancer emerged.

Many diseases are lumped under the denomination "cancer" because they share a fundamental biological feature, namely abnormal cell growth. However, cancer is not a single disease. It is a multiplicity of diseases caused by the uncontrolled growth of a single cell unleashed by mutations. Cancer cells can grow faster, flourish more profusely, adapt better, recover more rapidly, and repair faster... than normal cells. They are in effect more perfect versions of normal cells..... and can even become immortal. Therefore, we naively thought that cancer could be defeated by either preventing mutations from occurring in normal cells or else finding the means to eliminate the mutated cells without compromising normal growth. Unfortunately, this view does not take into account the perverse genetic intertwining of normal and cancerous growths. Cancer is woven into our genome as the mutated genes are but distorted versions of the normal ones; they are braided together and unbraiding ${ }^{1}$ them is the most formidable undertaking.

\section{Historical background-the $19^{\text {th }}$ century}

In the brief background that follows, I will not recount the past 4,000years of the history of cancer but rather begin with the important more recent years. In 1838, Matthias Schleiden and Theodor Schwann claimed that all living organisms were built out of fundamental building blocks called "cells", and that growth could occur either by "hypertrophy" (wherein the number of cells did not increase but each individual cell merely grew in size) or by "hyperplasia" (wherein, by contrast, growth was by virtue of the cells increasing in number). That view proved correct and still holds today. A number of hypotheses or theories of cancer were proposed since then.

\section{The blood suppuration hypothesis}

This is the first, albeit erroneous, hypothesis of the cause of cancer. In 1845, while treating a young slate-layer affected by what we now call leukemia, ${ }^{2}$ John Bennett characterized that illness as a "suppuration of blood" without actually finding the cause of infection at autopsy. In that same year, disbelieving Bennett's explanation, Rudolph Virchow doubted whether a blood abnormality might be the

${ }^{1}$ Changes in DNA that specifically affect genes and incite unlimited growth.

${ }^{2}$ Since the early 1900 s, it was clear that leukemia came in different forms: (a) chronic leukemia (CL): slow increase of white blood cells (WBC) until choking the bone marrow and spleen; (b) acute leukemia (AL) characterized by a rapid overgrowth of WBCs with two different subtypes according to the two types of WBCs (myeloid cells and lymphoid cells): (b1) acute myeloid leukemia (AML): and (b2) acute lymphoblastic leukemia (ALL), which are immature lymphoid cells. Cancers of more mature lymphoid cells are called lymphomas (L).

\author{
Alain L Fymat \\ International Institute of Medicine and Science, USA
}

Correspondence: Alain L Fymat, International Institute of Medicine and Science, California, USA, Tel (760) 485-9149, Email alain.fymat@fiimas.org

Received: November 28, 2016 | Published: November 30, 2016

cause of leukemia. His student, Michael Anton Biermer described the first known case of childhood leukemia in 1860. Leukemia was later described in the late 1880 s as a neoplasia..... Not a suppuration of blood.

In the late $19^{\text {th }}$ century, Percival Pott argued that cancer of the scrotum, endemic among chimney sweepers, was caused by chronic exposure to chimney soot and smoke. Also, in the 1890s, William Halsted successfully excised ("extirpated" was the word used then) single, localized solid tumors (as opposed to liquid tumors) such as solitary malignant lumps in the breast by radical mastectomy. Somewhat later, with the discoveries of X-rays by Wilhelm Roentgen in 1895, radioactivity by Henry Becquerel in 1898, and polonium and radium by Marie and Pierre Curie that same year, radiation (whether electromagnetic or nuclear) was also used as an adjunct to kill tumor cells localized in a single organ.

\section{The $20^{\text {th }}$ century}

In 1910, William H. Coley, James Ewing and Ernest Codman treated bone sarcoma with a mixture of bacterial toxins (the socalled "Coley's toxin") but with unpredictable results due probably to immune stimulation. Other hypotheses or theories were subsequently advocated.

\section{The somatic mutation hypothesis}

Very early in the $20^{\text {th }}$ century, in addition to the earlier observation of Pott regarding cancer of the scrotum, the only known causes of human cancers were environmental carcinogens (e.g., X-rays, radium) or organic chemicals (e.g., dye by-products) that were known to cause solid tumors. These observations led to the "somatic mutation hypothesis of cancer", which posited that environmental carcinogens (soot, smoke, radium, X-rays, etc.) permanently altered the cell and thus caused cancer. But the precise nature of the alteration was unknown and a more fundamental theory of carcinogenesis was still missing. 


\section{The viral origin theory}

In 1910, experimenting with spindle-cell sarcoma, Peyton Rous rather concluded that the cause of cancer was not a cell or an environmental carcinogen but some tiny particle within a cell...a virus, later called "Rous sarcoma virus" (RSV). As an endogenous causative factor, RSV dealt a deep blow to the exogenous somatic mutation theory.

\section{The infectious mononucleosis hypothesis}

In 1948, reinforcing Rous' conclusion of a virus as the cause of cancer, Denis Burkitt concluded that "Burkitt's lymphoma", an aggressive form of lymphoma, was caused by a human virus: the Epstein-Barr virus (EBV) that is responsible for infectious mononucleosis. The notion that cancer was an infectious disease was then resurrected (as hypothesized in the blood suppuration hypothesis), but this condemned the somatic mutation theory of cancer to its death. For approximately the next three decades, the viral origin of cancer then went dormant.

\section{The retroviral theory}

By the mid-1970s, however, a revival of the viral origin theory took place. Howard Temin had disproved the central dogma of molecular biology which posited a unidirectional flow of information: DNA $\rightarrow$ RNA (so-called transcription) $\rightarrow$ proteins. Rather, viruses possessed the reverse capacity: RNA $\rightarrow \mathrm{DNA} \rightarrow$ (so-called reverse transcription). Thus, a cancer-causing virus could become a physical part of a cell's genes. This and like viruses are known as retroviruses. The genes of retroviruses exist as RNA outside cells. When these RNA viruses infect cells, they make a DNA copy of their genes and attach this copy to the cell's genes. This DNA copy, called a provirus makes RNA copies, and the virus is regenerated to form new virus's adinfinitum, unleashing pathological mitosis, that is cancer. Unfortunately, as it turned out, retroviruses are not the cause of cancer but of another disease-HIV.

\section{The anti-vitamin theory and the war on cancer}

Following Rudolph Virchow's research on understanding the cause of leukemia, cancer was described as an "uncontrollable pathological cell division". Prior to the modern era of CT, MRI and other sophisticated imaging techniques, it was not possible to quantify cancer and measure its progression, the only exception being for leukemia. In leukemia, a simple blood draw and its analysis could inform on the white blood cell count and its changes as a result of drug treatment. This was the great contribution to the treatment of leukemia and beyond it of other cancers by Sidney Farber (of the famed DanaFarber Cancer Institute). Farber was kindled by two contemporaneous hematological findings; (a) George Minot's discovery that "pernicious" anemia was caused by a lack of vitamin $\mathrm{B}_{12}$ and could be treated by administrating that vitamin, and (b) Lucy Wills' treatment of "Indianworkers" anemia by the vitamin-like folic acid or folate (a crucial DNA building block). He was likewise kindled by the link between vitamins, bone marrow and normal blood. Unfortunately, neither of these two vitamins could treat cancer (actually, quite the contrary in the case of folic acid) so Farber searched for "anti-vitamins" (which do not exist). Nonetheless, this was the beginning of the search for a chemical to treat cancer that is "chemotherapy". The quest for a cure of leukemia took then the form of a military battle exemplified by Farber with the help of the legendary New York socialite Mary Lasker. Together, they launched the "War on Cancer"!

In the 1920s, while working for an antidote to the anemia of folate deficiency, Yellapragada Subbarao developed decoy molecular structures that nearly mimicked natural molecules. These structures can bind to enzymes and receptors and block their actions. They behave like antagonists to folic acid (anti-folates),... that is the very anti-vitamins Farber was looking for. In 1947-1948, Farber successfully treated, at least temporarily, a patient presenting with acute lymphoblastic leukemia (ALL) with the antifolate drug aminopterin supplied to him by Subbarao-an unprecedented remission in the history of leukemia. Unfortunately, the remission was short-lived (a few months) and the leukemia came roaring back. This could also truly be thought of as the beginning of chemotherapythe disappearance (even though temporary) of an aggressive systemic cancer via a chemical drug.

Two superficial theories were thus audaciously stitched together: cancer is caused by a virus and particular combinations of cytotoxic drugs would cure the cancer, which marked a new beginning of chemotherapy. It was only the latter theory that accounts for the success of chemotherapy, albeit limited in certain forms of cancer.

\section{The new beginning of chemotherapy}

In 1974, the survival rate from metastatic testes cancer was less than 5\%. With a three-drug cocktail called BVP (bleomycin, vinblastine, cisplatin abbreviated P for platinum), Larry Einhorn cured this solid cancer. The notion that even relatively indiscriminate cytotoxic agents discovered largely by accident would cure cancer captivated oncology. An avalanche of such drugs poured in: taxol, adriamycin, etoposide, bleomycin (an antibiotic), and an alphabet soup of other combinations (ABVD, BEP, C-POMP, ChlaVIP, $C H O P, A C T)$. At about the same time, using a high dose combination chemotherapy (a cocktail of seven drugs), Ian Magrath and John Ziegler cured Burkitt's lymphoma. Yet, despite the escalation of drugs and doses, the efficacy of the drug regimen remained minimal. The pattern repeated itself regularly for many forms of cancer.

\section{The endogenous proto-oncogene theory}

Also in the mid-1970s, it was Harold Varmus and J. Michael Bishop who showed that a precursor of a cancer-causing gene-which they called the "proto-oncogene", was a normal cellular gene existing inside cancer cells. Mutations induced by chemicals or X-rays caused cancer, not by inserting foreign genes, but by activating such endogenous proto-oncogenes. Thus, cancer genes come from within the human genome.

\section{The two-hit hypothesis-proto-oncogenes and tumor suppressor genes}

In the early 1970s, Alfred Knudson proposed the two-hit hypothesis of cancer, initially for retinoblastoma. Retinoblastoma has distinct variants, an inherited "familial" form and a "sporadic" form. In the former form, cancer's onset is rapid with diagnostic typically 2-6months after birth whereas, in the latter form, it typically appears 2-4years after birth. Further, in the familial form of retinoblastoma, only one genetic change is required to develop the cancer whereas two genetic changes are required in the sporadic form. Further, Knudson proposed that genes come in two flavors: "positive" genes that are mutant-activated versions of normal cellular genes (they accelerate cell division, but only when the cell receives an appropriate growth signal) and "negative" genes that suppress cell division when the cell receives appropriate signals, having been inactivated by mutations. Both abnormalities activate proto-oncogenes and inactivate tumorsuppressors. When this activation/inactivation falls out of equilibrium, cancer then develops. 
Over the decades that followed, a large array of drugs were synthesized for the fight on cancer. Hodgkin's disease is now cured with multi-drug chemotherapy; locally advanced lung cancer is controlled with the triadic treatment (surgery+chemotherapy+radiation); lymphoblastic leukemia can be induced into a prolonged remission after intensive chemotherapy with cure rates of $80 \%$ routinely achieved. The mortality for every form of cancer (lung, breast, colon, prostate, etc.) has continuously dropped for fifteen straight years. For some other cancers (colon, cervix), the decline is almost certainly due to the success of secondary prevention-cancer screening. For leukemia, lymphoma, and testicular cancer, the decline is the result of successful chemotherapeutic treatments. The death rate for breast cancer has been dramatically brought down by the combination (mammography+surgery+adjuvant chemotherapy).

\section{Conclusion}

In summary, after a circuitous detour through several hypotheses and theories (elements of which are nonetheless valuable), we have now come to the conclusion that cancer is stitched into our genome; oncogenes arise from mutations in essential genes that regulate the growth of cells; mutations accumulate in these genes when DNA is damaged by carcinogens (theoretically preventable), but also by seemingly random errors in copying genes when cells divide (a flaw deeply entrenched in ourselves and therefore unpreventable). Thus, we can rid ourselves of cancer only in as much as we can rid ourselves of the processes in our physiology that depend on growthaging, regeneration, healing, and reproduction. The best treatment remains prevention, at least the minimization of those epigenetic and ecogenetic factors that trigger the expression of cancer.

With the momentous advances of the Human Genome Project (HGP) and the sequel the Human Cancer Genome Project (HCGP), we have now come to the realization that understanding more intimately the biology of cancer, gene by gene and pathway by pathway, will direct us into the right direction for cancer therapeutics. A second direction would obviously be cancer prevention, or at least prevention of those factors that may trigger cancer. The third and last direction would be to integrate our understanding of aberrant genes and pathways to explain the behavior of cancer.

\section{Acknowledgments}

None.

\section{Conflicts of interest}

Author declares there are no conflicts of interest. 